\title{
Clima ambientale e clima emotivo: due pilastri evolutivi poco tangibili ma tremendamente importanti per la nostra vita
}

\author{
David Mariani ${ }^{1}$, Alessandro Capitanini ${ }^{2}$ \\ ${ }^{1}$ Healthy Habits Academy Director, Montecatini Terme, Pistoia - Italia \\ ${ }^{2} \mathrm{SOC}$ Nefrologia e Dialisi Ospedale di Pistoia, Azienda Sanitaria Toscana Centro, Pistoia - Italia
}

\begin{abstract}
Environmental climate and emotional climate: two evolutionary pillars that are not very tangible but tremendously important for our life

Our evolutionary history is based on four fundamental pillars: physical activity, nutrition, inner mood and external environment. We live at the rhythm of an inner clock that leads everything we do, the so called circadian rhythm. The circadian system, that is ubiquitous across species, generates $\sim 24 \mathrm{~h}$ rhythms in virtually all biological processes, and allows them to anticipate and adapt to the $24 \mathrm{~h}$ day/night cycle, thus ensuring optimal physiological function. During the day our organism spends its energy in order to complete catabolic pathways which help us in our relationship with the external environment, while at night anabolic pathways, repairing, strengthening and growth-orientated, prevails. External environment can influence both our circadian rhythm and our body chemistry. Within the last 100 years, environmental conditions are extremely changed: the artificial light (also by televisions screens, smartphones and computers) is lighting up our world 24 hours a day, the background noise is a constant in our everyday life and the air pollution represent risk factors for our health and well-being by causing or facilitating diseases. Also our inner mood, emotions and environment are crucial for human beings' and planet's health and for the future of mankind.
\end{abstract}

Keywords: Circadian rhythms, Light pollution, Noise pollution, Air pollution, Environment, Health hazard

Dopo aver visto l'importanza dei primi due pilastri evolutivi (attività fisica e nutrizione), ovverosia del movimento per trovare cibo, vediamo insieme quali sono gli altri due elementi in grado di incidere in modo decisivo sulla nostra salute: ambiente e stato emotivo.

Entrambi, infatti, contribuiscono a modificare rapidamente le condizioni di equilibrio della chimica organica umana $(1,2)$. Come sempre, dobbiamo essere capaci di rivalutare criticamente la nostra evoluzione per cercare di capire qual è il miglior uso che possiamo fare di questi due pilastri.

II sistema circadiano, che è ubiquitario tra le specie, genera ritmi di $\sim 24$ ore in quasi tutti i processi biologici e consente di anticipare e adattarsi al ciclo giorno/notte di 24

Received: December 12, 2019

Accepted: December 31, 2019

Published online: February 20, 2020

Indirizzo per la corrispondenza:

Healthy Habits Academy

Via Bacci 21

51016 Montecatini Terme, Pistoia Italia

d.mariani@healthyhabits.it ore, garantendo così una funzione fisiologica ottimale. Per millenni l'essere umano ha vissuto nel rispetto dei cicli circadiani: giorno e notte, insieme alle stagioni, dettavano i ritmi della vita; i rumori presenti erano quelli della natura, degli animali, della voce umana; la vita si svolgeva in gran parte all'aria aperta.

Il consumo di cibo e la sete, l'attività locomotoria, il sonno e la veglia, le funzioni intestinali, la pressione sanguigna, i vari metabolismi, la temperatura corporea e altri elementi hanno dei ritmi precisi (3).

Nel suo trattato sulle erbe del 1632, John Wren descrisse i flussi quotidiani dei quattro umori-fluidi e il loro ritmo e ipotizzò quelli che sono i concetti moderni della neurobiologia circadiana, ovvero la presenza di un ordine inesorabile e ripetitivo delle nostre vite interne che governa i cicli quotidiani dello stato d'animo, dell'intelletto e delle abilità fisiche (4).

Nell'uomo, il ritmo circadiano più ovvio è il ciclo del sonno e della veglia. Questo ritmo è fondamentale se si pensa che durante il giorno la nostra fisiologia è dedicata prevalentemente ai processi catabolici per facilitare l'impegno con l'ambiente; di notte, viceversa, predominano le funzioni anaboliche, ovvero dedicate a crescita, riparazione e consolidamento. 
Questi programmi contenuti nel nostro DNA stabiliscono un ordine temporale interno che ottimizza la nostra fisiologia. Al calare della sera, la nostra temperatura corporea si riduce e la melatonina viene secreta dall'epifisi per facilitare il sonno. Ma l'inizio del sonno è accompagnato anche dall'aumento della secrezione dell'ormone della crescita e della prolattina; poco prima dell'alba l'attivazione dell'asse adrenocorticotropico ci prepara invece alle esigenze fisiche e mentali del risveglio.

In poche parole, la nostra organizzazione fisiologica è necessaria e anticipatoria per fronteggiare le diverse situazioni della vita, permettendoci di assolvere al meglio i compiti dei vari e diversi possibili momenti della giornata o della notte, per sostenere il massimo sforzo o il riposo.

Il sistema circadiano di molte specie è sensibile ai cambiamenti stagionali nel ciclo naturale luce-buio. Ad esempio, i criceti e le pecore mostrano una durata dei livelli elevati di melatonina più lunga in inverno che in estate, i quali, a loro volta, influenzano i cambiamenti stagionali di: stato riproduttivo, colore del mantello e/o aumento di peso (5-7). L'evoluzione del nostro genoma non è diversa da quella di molti animali e il nostro interruttore è rappresentato dalla luce.

L'invenzione della luce artificiale, insieme all'industrializzazione, hanno modificato completamente il nostro ambiente di vita negli ultimi 150 anni.

Attualmente, nei Paesi sviluppati, le notti sono eccessivamente illuminate (luce di notte), mentre il giorno è principalmente trascorso al chiuso, e quindi le persone sono esposte a intensità di luce molto inferiori rispetto a quelle naturali di giorno e viceversa di notte. Nonostante l'impatto positivo della luce artificiale (non sarebbe più possibile vivere senza!), stiamo pagando un caro prezzo per il facile accesso alla luce durante la notte. Purtroppo, la disorganizzazione del nostro sistema circadiano, detta chronodisruption (CD), legata alle perturbazioni nel ritmo della melatonina, è associata a un'aumentata incidenza di diabete, obesità, malattie cardiache, deterioramento cognitivo e affettivo, invecchiamento precoce e di alcuni tipi di cancro. La relazione tra fotorecettori della retina e melanopsina in alcune cellule gangliari dimostra che l'intensità della luce, i tempi e il suo spettro sono fondamentali per mantenere l'orologio biologico correttamente funzionante (8-13). Risulta importante anche lo spettro della luce: la luce con forti componenti bianco-blu, emesse dalla luce a LED e dai dispositivi elettronici (tv, computer e smartphone), rappresenta un pericolo per la nostra salute e ne andrebbe limitata l'esposizione (14).

Chi abita negli agglomerati urbani è sottoposto a una sorta di inquinamento acustico costante: come la luce, anche il rumore è il frutto della rivoluzione industriale. Questo rumore di fondo continuo, a bassa informazione, a differenza del rumore naturale, favorente momenti di riflessione e tranquillità, è in grado di provocare danni alla nostra salute e il Times di
Londra ha addirittura definito il rumore "la peggiore piaga della vita contemporanea".

Gli effetti fisiologici del rumore ambientale sull'uomo sono ben documentati (15-17). Babisch ha riassunto gli effetti dello stress del rumore come segue: "Il rumore attiva l'asse ipofisi-corticale-surrenale e l'asse simpatico-midollare-surrenale, con alterazione (aumento) degli ormoni dello stress tra cui adrenalina, noradrenalina e cortisolo». Ciò è comprovato sperimentalmente oramai da tempo sia in corso di rumore acuto che cronico. Questi ormoni (definiti ormoni dello stress) sono utili se liberati al momento opportuno ma dannosi se liberati in modo continuo (18).

Altro capitolo fondamentale del pilastro ambiente è quello che concerne la qualità dell'aria. Questa è altrettanto importante rispetto agli alimenti che mangiamo perché, oltre a influenzare direttamente la salute dell'essere umano, riflette anche lo stato degli ecosistemi $(19,20)$.

L'inquinamento atmosferico causa fino a 7 milioni di morti premature all'anno e un numero ancora maggiore di ricoveri e inabilità per malattia. Negli ultimi 30 anni, numerosi studi hanno dimostrato che l'inquinamento atmosferico è causa di un aumento della morbilità e mortalità cardiovascolare e respiratoria sia negli adulti che nei bambini in tutto il mondo (21-24). Si pensi che l'inquinamento atmosferico è stato anche associato all'aumento del rischio di complicanze in gravidanza $(25,26)$.

Sorprendentemente il $60 \%$ di queste morti è attribuibile all'inquinamento delle case o dei luoghi di lavoro, ove inquinanti atmosferici interni sono: fumo di sigaretta, prodotti del tabacco e altri processi che rilasciano gas o particelle (come i gas derivanti dai combustibili usati nella cottura o nel riscaldamento) e, molto importante, la scarsa ventilazione intesa come ricambio d'aria. A quest'ultimo proposito, nel mondo "occidentale", i sistemi di condizionamento dell'aria, l'esposizione alla formaldeide, l'esposizione passiva al fumo di sigaretta e gli edifici sempre più sigillati contribuiscono in modo specifico ad aumentare i livelli di inquinamento dell'aria interna $(27,28)$.

Respirare all'aria aperta, avere momenti di silenzio nella natura e riposare profondamente nelle ore notturne sono situazioni indispensabili al mantenimento dell'equilibrio del nostro organismo. Sono queste situazioni, infatti, che attivano il sistema nervoso parasimpatico, inducendo rilassamento e contrastando l'eccesso di stress (da iperattivazione del sistema nervoso simpatico): erano le attività associate al tempo di pace nella nostra vita primordiale.

Se il clima ambientale è fondamentale, altrettanto lo è il nostro clima emozionale.

Giocare, coccolarsi, difendersi, consolarsi e curarsi reciprocamente sono abilità sviluppate in millenni di storia evolutiva che sono incise in modo indelebile nel nostro sistema limbico: queste situazioni sono in grado tutt'oggi di modificare significativamente la secrezione di molti ormoni.

Siamo animali sociali e abbiamo bisogno degli altri per i motivi sopracitati $(29,30)$. 
La civiltà moderna e l'evoluzione (se così possiamo chiamarla) ci hanno portato, anche in questo caso, ad abbandonare sempre più le abitudini primordiali. La vita di oggi ci spinge all'abbandono delle relazioni fisiche e del contatto umano. Anche la medicina, con l'enorme costante progresso tecnologico-scientifico, prevede sempre meno tempo di relazione e contatto tra medico e paziente. Oggi sappiamo con certezza scientifica che il contatto umano, le parole, la compassione possono attivare nella mente di un paziente processi biochimici identici a quelli di un farmaco antidolorifico (31). II sovraccarico tecnologico della medicina purtroppo ha drammaticamente coinciso con un impoverimento antropologico, con diminuzione della sua dimensione umana e solidaristica, e soprattutto provocato un progressivo distacco del medico dal paziente. L'empatia e le emozioni sono il nutrimento del benessere: saper e sapersi perdonare si sono rivelate condizioni di grande vantaggio per chi riesce a farlo. La scienza ha dimostrato che saper perdonare ci migliora la vita e molto probabilmente ce la allunga (32).

Come sempre, la storia della nostra evoluzione ci fornirebbe semplici soluzioni a problemi che ci paiono complessi.

Non possiamo permetterci, come abbiamo fatto negli ultimi cento anni, di modificare troppo velocemente le nostre abitudini e l'ambiente in cui viviamo, perché il nostro genoma non può riuscire ad adattarvisi cosi velocemente senza andare incontro a disturbi e malattie.

Per cercare di vivere felici e in salute abbiamo bisogno di persone e non di strumenti... almeno per qualche altro millennio. Per pacificare l'anima abbiamo bisogno dell'amore dei nostri simili e per lenire il dolore e le preoccupazioni abbiamo bisogno di conforto di altri esseri umani. Conoscere la nostra evoluzione e quella delle abitudini radicate dentro di noi sarà un meccanismo di consapevolezza indispensabile da trasmettere alle prossime generazioni per evitare errori di percorso fatali. Non è mai troppo tardi per migliorare.

\section{Disclosures}

Financial support: No financial support was received for this submission.

Conflict of interest: The authors have no conflict of interest.

\section{Bibliografia}

1. Ulrich RS. View through a window may influence recovery from surgery. Science. 1984;224:420-1.

2. Health Council of the Netherlands and Dutch Advisory Council for Research on Spatial Planning, Nature and the Environment. Nature and health. The influence of nature on social, psychological and physical well-being. The Hague: Health Council of the Netherlands and RMNO 2004. Available at: http:// www.gezondheidsraad.nl/en/publications/nature-and-healthinfluence-nature-social-psychological-and-physical-well-being

3. Curtis AM, Fitzgerald GA. Central and peripheral clocks in cardiovascular and metabolic function. Ann Med. 2006;38:552-9.

4. Hastings $\mathrm{MH}$, Reddy $\mathrm{AB}$, Maywood ES. A clockwork web: circadian timing in brain and periphery, in health and disease. Nat Rev Neurosci. 2003;4(8),649-61.
5. Brainard GC, Petterborg LJ, Richardson BA, Reiter RJ. Pineal melatonin in syrian hamsters: circadian and seasonal rhythms in animals maintained under laboratory and natural conditions. Neuroendocrinology. 1982;35:342-8.

6. Carr AJ, Johnston JD, Semikhodskii AG, Nolan T, Cagampang FR, Stirland JA, Loudon AS. Photoperiod differentially regulates circadian oscillators in central and peripheral tissues of the syrian hamster. Curr Biol. 2003;13:1543-8.

7. Dardente $H$, Wyse CA, Birnie MJ, Dupré SM, Loudon AS, Lincoln GA, Hazlerigg DG. A molecular switch for photoperiod responsiveness in mammals. Curr Biol. 2010;20:2193-8.

8. Haim A, Portnov BA. Light Pollution as a New Risk Factor for Human Breast and Prostate Cancers. Dordrecht: Springer Netherlands; The Netherlands 2013.

9. De Beaune SA, White R. Ice Age Lamps. Sci Am. 1993;206: 108-13.

10. Nordhaus WD, Nordhaus WD. Do real-output and real wage measures capture reality? The history of lighting suggests not. In: Bresnahan TF, Gordon RJ, eds. The Economics of New Goods. Chicago, IL, USA: The University of Chicago Press 1994:27-70.

11. Cinzano P, Falchi F, Elvidge $C D$. The first world atlas of the artificial night sky brightness. Monthly Notices of the Royal Astronomical Society 2001;328:689-707.

12. Navara KJ, Nelson RJ. The dark side of light at night: Physiological, epidemiological, and ecological consequences. J Pineal Res. 2007;43:215-24.

13. Pauley SM. Lighting for the human circadian clock: Recent research indicates that lighting has become a public health issue. Med Hypotheses. 2004;63:588-96.

14. Renard G, Leid J. The dangers of blue light: True story! J Fr Ophtalmol. 2016;39:483-8.

15. Babisch W. Cardiovascular effects of noise. Noise Health. 2011; 13:201-4.

16. Babisch W. Stress hormones in the research on cardiovascular effect of noise. Noise Health. 2003;5:1-11.

17. Jarup L, Dudley ML, Babisch W; HYENA Consortium. Hypertension and Exposure to Noise near Airports (HYENA): study design and noise exposure assessment. Environ Health Perspect. 2005;113(11):1473-8.

18. Babisch W. The noise/stress concept, risk assessment and research needs. Noise Health. 2002;4:1-11.

19. WHO. Review of evidence on health aspects of air pollutionREVIHAAP project: technical report. Copenhagen: WHO Regional Office for Europe 2013.

20. Haase D, Larondelle N, Andersson E, et al. A quantitative review of urban ecosystem service assessments: concepts, models, and implementation. Ambio. 2014;43(4):413-33.

21. Fang Y, Naik V, Horowitz LW, Mauzerall DL. Air pollution and associated human mortality: the role of air pollutant emissions, climate change and methane concentration increases from the preindustrial period to present. Atmos Chem Phys. 2013; 13(3):1377-94.

22. Bernstein JA, Alexis N, Barnes C, et al. Health effects of air pollution. J Allergy Clin Immunol. 2004;114:1116-23.

23. Dockery DW. Epidemiologic evidence of cardiovascular effects of particulate air pollution. Environ Health Perspect. 2001;109 (Suppl 4):S483-6.

24. Patel MM, Miller RL. Air pollution and childhood asthma: recent advances and future directions. Curr Opin Pediatr. 2009; 21:235-42.

25. Dockery DW, Pope CA 3rd. Acute respiratory effects of particulate air pollution. Annu Rev Public Health. 1994;15:107-32.

26. Choi H, Rauh V, Garfinkel R, Tu Y, Perera FP. Prenatal exposure to airborne polycyclic aromatic hydrocarbons and risk of intrauterine growth restriction. Environ Health Perspect. 2008;116:658-65. 
27. Smith KR, Samet JM, Romieu I, Bruce N. Indoor air pollution in developing countries and acute lower respiratory infections in children. Thorax. 2000;55:518-32.

28. Yu BF, Hu ZB, Liu M, Yang HL, Kong QX, Liu YH. Review of research on air-conditioning systems and indoor air quality control for human health. Int J Refrig. 2009;32:3-20.

29. Jerath R, Crawford MW. How Does the Body Affect the Mind? Role of Cardiorespiratory Coherence in the Spectrum of Emotions. Adv Mind Body Med. 2015 Fall;29(4):4-16.
30. Decety J, Norman GJ, Berntson GG, Cacioppo JT. A neurobehavioral evolutionary perspective on the mechanisms underlying empathy. Progr Neurobiol. 2012;98(1):38-48.

31. Evers AWM, Colloca L, Blease C, et al. Implications of Placebo and Nocebo Effects for Clinical Practice: Expert Consensus. Psychother Psychosom. 2018;87(4):204-10.

32. Toussaint LL, Owen AD, Cheadle A. Forgive to live: forgiveness, health, and longevity. J Behav Med. 2012;35(4):375-86. 